\title{
The elusive seven-membered cyclic imino ether tetrahydrooxazepine
}

Bart Verbraeken, ${ }^{\mathrm{a} \dagger}$ Jan Hullaert, ${ }^{\mathrm{b} \dagger}$ Joachim van Guyse, ${ }^{\mathrm{a}}$ Kristof Van Hecke, ${ }^{\mathrm{c}}$ Johan Winne, ${ }^{\mathrm{b} *}$ Richard Hoogenboom $^{\mathrm{a} *}$

aSupramolecular Chemistry group, Centre of Macromolecular Chemistry (CMaC), Department of Organic and Macromolecular Chemistry, Ghent University, Krijgslaan 281 S4, 9000 Ghent, Belgium

${ }^{b}$ Organic Synthesis group, Department of Organic and Macromolecular Chemistry, Ghent University, Krijgslaan 281 S4, 9000 Ghent, Belgium

${ }^{c}$ XStruct, Department of Chemistry, Ghent University, Krijgslaan 281 S3, 9000 Ghent, Belgium

Supporting Information Placeholder

ABSTRACT: Cyclic imino ether heterocycles are used as ligands in transition metal catalysis, in various drugs and as reactive monomers in living cationic ring-opening polymerization (CROP). While five- and six-membered cyclic imino ethers, i.e. 2-oxazolines and 4,5-dihydro-1,3-oxazines, have extensively been studied in these areas, their seven-membered ring counterparts have remained unexplored. Herein, we report the synthesis of 2-phenyl-4,5,6,7-tetrahydro-1,3-oxazepine allowing reassignment of the earlier, incorrectly reported 4,5,6,7-tetrahydro-1,3-oxazepines as their $N$-acylated pyrrolidine isomers. Finally, we also report a comparison of the CROP reactivity of a homologous series of cyclic imino ethers with a 2carbon, 3-carbon and 4-carbon methylene bridge, revealing a remarkable ring size effect.

Imino ethers, corresponding to the bonding arrangement $\mathrm{R}^{1}-\mathrm{N}=\mathrm{CR}^{2}-\mathrm{O}-\mathrm{R}^{3}$ can be regarded as $O$-alkylated versions of the thermodynamically less favorable imidic acid tautomer of an amide moiety. Therefore, imino ethers can be considered as 'spring loaded' reagents, and their spontaneous rearrangement to form a tertiary amide isomer (via a formal 1,3-alkyl shift) is a classical organic reaction with a high thermodynamic driving force. ${ }^{1}$ The most studied cyclic imino ethers (CIE) are the 2-oxazolines (4,5-dihydro-1,3oxazoles), comprising a five-membered ring. 2-Oxazolines are widely used in asymmetric catalysis as chiral ligands, for a broad range of different reactions such as hetero Diels-Alder reactions, ${ }^{2}$ aldol condensations ${ }^{3}$ and Michael additions. ${ }^{4}$ Besides their ability to act as ligands in catalysis, 2-oxazolines can also be found as core scaffolds in bioactive molecules ranging from antibacterials, ${ }^{5}$ antifungals ${ }^{6}$ to antituberculosis agents. ${ }^{7}$ Furthermore, 2-oxazolines can also be applied as an efficient protective group for carboxylic acids, with a tolerance to harsh (basic) reaction conditions e.g. Grignard and hydrides. ${ }^{8}$ Finally, 2oxazolines can be used as monomers in living cationic ring opening polymerization (CROP). ${ }^{1}$

The polymerization behavior of 2-oxazolines can be related to its intrinsic nucleophilic and electrophilic properties. Imino ethers are relatively good nucleophiles, and the CROP of 2-oxazolines can be readily 
induced by strong alkylating reagents, leading to a highly electrophilic oxazolinium-type cation that constitutes a highly reactive chain end to which subsequent monomers can add, resulting in the controlled synthesis of poly(2-alkyl/aryl-2-oxazoline)s (PAOx) and related macromolecules (Scheme 1a). ${ }^{9}{ }^{10}$ Due to their water-soluble nature, these tertiary amide containing polypeptide-like polymers are actively explored for biomedical applications. ${ }^{11-14} \mathrm{CROP}$ of CIEs also serves as a versatile platform to prepare various macromolecular architectures. ${ }^{1,15,16}$ In contrast to classical ring-opening polymerizations, like those of cyclic ethers and esters, the main driving force for the polymerization is not the release of ring strain, but rather the concurrent isomerization of the imino ether function to a thermodynamically more stable amide group. Therefore, it may be anticipated that the CROP of CIE should be favorable, regardless of monomer ring size. Even though the vast majority of studies of CROP of CIEs have focused on 2-oxazolines, the homologous and relatively 'strain-free' 5,6-dihydro-4H-1,3-oxazines (2-DHOx) have also been successfully polymerized yielding polymers with markedly different properties than those based on the 5-membered ring CIEs (Scheme 1a). ${ }^{17-22}$

Scheme 1. Cationic ring-opening polymerization (CROP) of cyclic imino ethers: A. established processes and monomer classes, $B$. new monomer class reported herein.

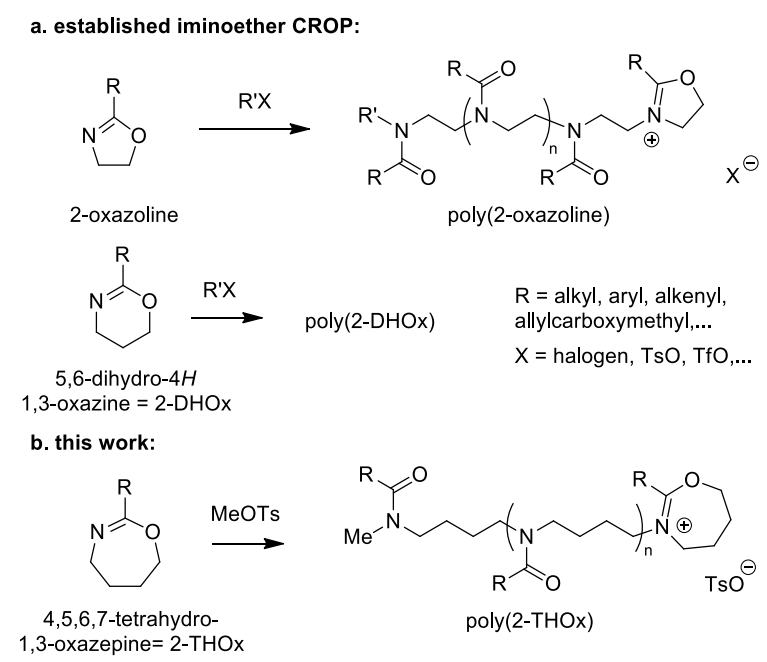

To the best of our knowledge, and somewhat unexpectedly, the homologous 4,5,6,7,-tetrahydro-1,3oxazepine (2-THOx) monomers nor any of the larger rings sizes have ever been studied for CROP. By further increasing the ring-size, resulting in an addition of an extra methylene in the polymer backbone, it may be expected that the water solubility and glass transition temperature of the resulting polymers will be lower compared to analogous poly(2-oxazoline)s and poly(2-DHOx)s. ${ }^{17}$ Furthermore, the poly(2THOx) may serve as precursor for making linear polybutylenimine. In 1996, Miyamoto et al. reported a single example of a related CROP of a seven-membered cyclic carbamimidate, ${ }^{23}$ leading to a polyureatype polymer, indicating the feasibility of such a polymerization also for CIEs monomers. This apparent 
hiatus in the literature on CIE CROP may be related to the fact that the synthesis of 2-THOx is also only sparingly reported in the literature, and these compounds are thus not readily available as monomers. ${ }^{24-29}$

Intrigued by the possibility to introduce a four-carbon linker between the repeating amide units, we set out to investigate the polymerization of such seven-membered CIE monomers. A suitable monomer was purportedly found in the literature in the form of 2-phenyl-4,5,6,7-tetrahydro-1,3-oxazepine (2-phenyl-2THOx) 1, the synthesis of which had been described in four independent literature reports using two different methods (Scheme 2). ${ }^{24-29}$

However, the 2-phenyl-2-THOx 1 prepared according to the literature procedure reported by Mollo et al. ${ }^{29}$ did not have the expected physical and chemical properties, based on our experience with 2-oxazolines and 2-DHOx. The obtained product showed a complete lack of any kind of reactivity in CROP, and even any type of alkylation reactivity. Having excluded possible causes and/or contaminations for this unexpected lack of reactivity, we rapidly arrived at a critical reassessment of the structure assigned to the material we obtained, as its analytical NMR spectroscopic data showed significant discrepancies with those typical of 2-DHOx or 2-oxazoline monomers, especially the protons of the methylene groups adjacent to the imino ether group in the ring (SI Figures S1 and S2). In fact, the NMR data seemed to be more in line with that typical of the resulting CIE polymers, indicating the presence of an amide bonding arrangement (Scheme 2).

Scheme 2. Reported synthesis for the 2-phenyl-4,5,6,7-tetrahydro-1,3-oxazepine 1, and the observed benzoylated pyrrolidine outcome (top); Confirmation of revised product assignment via alternative synthesis of the proposed amide 2 (bottom).

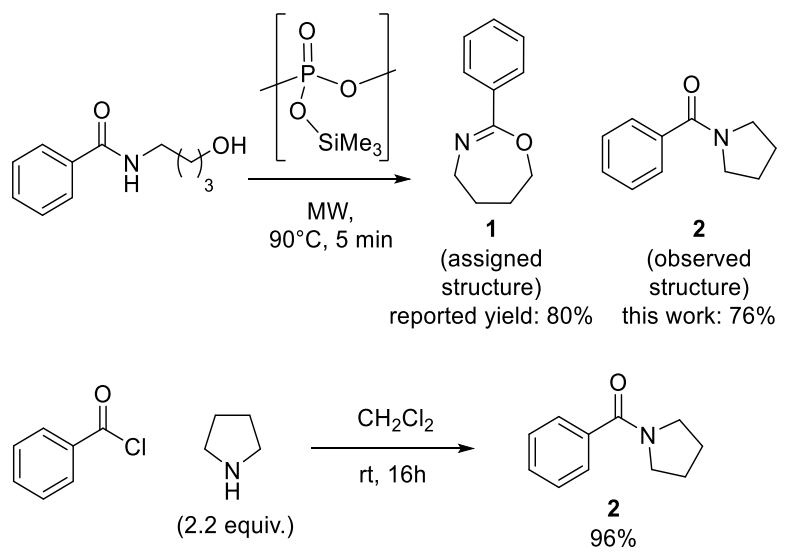

In order to confirm our reassignment, we prepared the isomeric amide 2 by benzoylation of pyrrolidine, and found this material to be indistinguishable to the previously obtained compound, also showing ${ }^{1} \mathrm{H}$ and ${ }^{13} \mathrm{C}$ NMR data completely consistent with literature data reported for the compound mistakingly assigned in literature to the structure of 2-phenyl-2-THOx 1. ${ }^{24-29} \mathrm{~A}$ careful analysis, and comparison with their $\mathrm{N}$ acylated pyrrolidine analogues, of all literature data that was reported for 4,5,6,7-unsubstituted-2-THOx, (SI table S1 to table S11) then led us to the remarkable conclusion that the parent heterocyclic structure, 
a non-ring fused seven membered CIE, has in fact never been prepared, and that all previously claimed reports are based on a misassignment of the (rearranged) amide isomer, i.e. the $N$-acylated pyrrolidine ring system rather than the claimed CIE structure. We speculate that these anomalous cyclizations proceeds through an endothermic amide-iminol tautomerization, followed by a rapid intramolecular alkylation (see SI for further discussion).

In order to confirm the above assignment, and in order to achieve our initial goal of investigating the CROP of 2-THOx-type monomers, we set out to extend the homologous series of 2-oxazoline and 2DHOx type monomers by developing the first synthesis of a 2-THOx monomer (Scheme 3). As the direct synthesis of the 2-THOx ring by cyclization is not feasible, we reasoned that the known cyclic carbamate 3 would be an ideal starting point for our purpose, as 2-oxazepanones are a robust and very well established heterocyclic product class. ${ }^{23,30,31}$ Furthermore, we expected to be able to convert this cyclic carbamate into the desired 2-THOx under relatively mild conditions, hopefully preventing thermal and/or (Lewis) acid-catalyzed imino ether rearrangement into the more stable amide. Selective $O$-alkylation of 3 was achieved using Meerwein's salt following a procedure reported by Miyamoto et al. ${ }^{23}$ In a second step, also inspired by related chemistry developed by Miyamoto to prepare seven-membered cyclic carbamimidates, ${ }^{23}$ a simple addition/elimination-type reaction with a range of organometallic reagents was explored aiming at a substitution of the exocylic ethoxy moiety by a carbon-nucleophile, which would directly result in the elusive seven-membered 2-THOx-type monomer. Although simple alkyllithium nucleophiles such as $n$-butyllithium proved to be troublesome in this scheme, the use of aryllithium reagents gave a reasonably clean conversion to the desired 2-aryl-2-THOx's $\mathbf{1}, \mathbf{5 a}$ and $\mathbf{5 b}$, which could all be obtained in pure form through column chromatography. The monomer 1 was obtained in a moderate, but useful $41 \%$ yield, and could be scaled to multi-gram scale. Two other 2-(hetero)aryl-2-THOx's were obtained in similar yield on a smaller scale. Compound 1 crystallized upon storage in the freezer, allowing an unambiguous structure assignment via X-ray diffraction analysis of a single crystal of this compound (Scheme 3). In addition, the crystal structures of 2-THOx's $\mathbf{5 a}$ and $\mathbf{5 b}$ were also determined (see SI).

Scheme 3. Synthesis of 2-aryl-4,5,6,7-tetrahydro-1,3-oxazepines and molecular structure of 1 as determined by single crystal X-ray diffraction analysis (SI Figure S20).

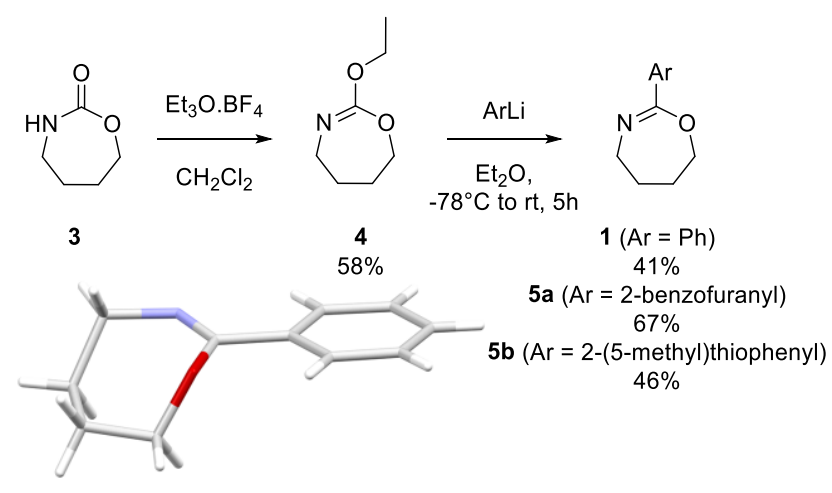


Having established the first synthetic entry to a 4,5,6,7-unsubstituted-2-tetrahydrooxazepine, suitable for CROP, we next examined the polymerization behavior of this novel CIE monomer and compared it with 2-phenyl-2-oxazoline 6 and 2-phenyl-2-DHOx 7. Because of the relative dearth of material, the polymerization was monitored by high temperature $\left(120{ }^{\circ} \mathrm{C}\right)$ on-line ${ }^{1} \mathrm{H}$ NMR analysis in a concentrated solution in chlorobenzene, a solvent expected to be able to dissolve all three monomers and their respective polymers. As initiator, methyl tosylate was added in a 1:100 ratio with respect to the monomer, which would result in a DP of 100 at full conversion. The propagation rate constant $\left(k_{p}\right)$ determined for 6 from kinetic experiments in chlorobenzene and in $d 3$-acetonitrile are very similar, allowing a reference to the literature data that mainly uses this solvent. Note that the decrease in polymerization rate of 6 at high conversion in $d_{3}$-acetonitrile can be ascribed to the high viscosity of the obtained polymer solution leading to diffusion limited monomer addition. As expected based on previous reports, ${ }^{19}$ the homologous monomer 7 of the 2-DHOx-type showed a considerably slower propagation with a $k_{p}$ that is almost 10 times lower than that of the 2-oxazoline 6. For the new monomer $\mathbf{1}$, however, the polymerization proved exceedingly slow at $120{ }^{\circ} \mathrm{C}$, and had to be monitored over the course of several days. Nevertheless, by plotting the relative integrations of the well-resolved resonances for the monomer and the poly(2-THOx) oligomers, we could determine a $\mathrm{k}_{\mathrm{p}}$-value of $1.27 \times 10^{-5} \mathrm{~mol} \mathrm{~L} \mathrm{~L}^{-1} \cdot \mathrm{s}^{-1}$, which is 540 times slower than the 2oxazoline monomer 6, and 60 times slower that the 2-DHOx monomer 7 under the same conditions. After termination with $1 \mathrm{~mol} / \mathrm{L} \mathrm{KOH}$ solution in methanol, we analyzed the reaction mixture by MALDI TOFMS, showing the expected oligo(2-THOx) distribution with a maximum intensity peak corresponding to a DP of 7 and 8, in line with the observed monomer conversion in the final ${ }^{1} \mathrm{H}$ NMR spectra $($ i.e. $7.6 \%$ monomer conversion, see SI figure S14). Furthermore, the parent ion could be detected in MALDI-TOF MS, but further in depth studies will be performed in the near future to evaluate the living nature of the polymerization.
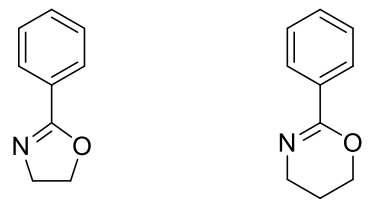

7

6
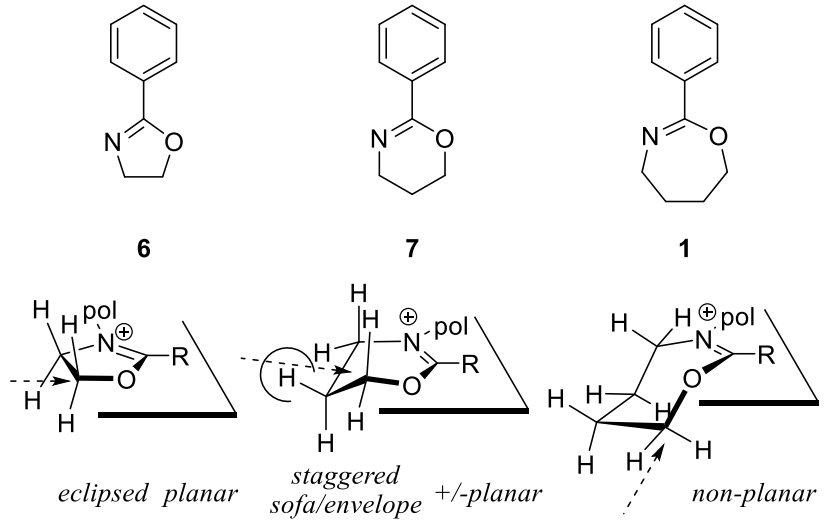


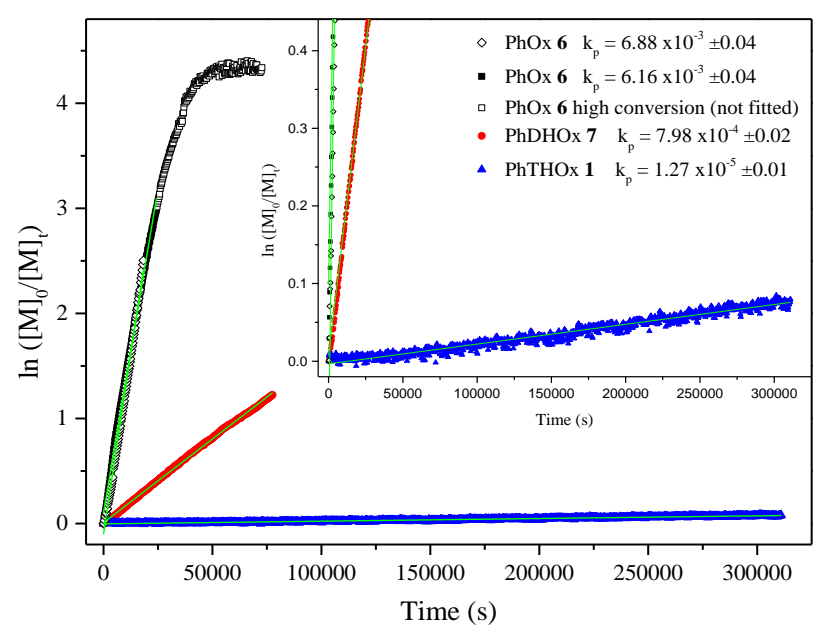

Figure 1. Structures, reactive chain end conformations, and kinetic plots for polymerization of the homologous series of monomers 6, 7 and $\mathbf{1}$. Reactions were performed in chlorobenzene at $2 \mathrm{~mol} / \mathrm{L}$ monomer concentration with methyl tosylate as initiator heated in the NMR spectrometer to $120^{\circ} \mathrm{C}(\mathrm{DP}=100)$. The exact polymerization conditions can be found in the SI (5). 2-phenyl-2-oxazoline (PhOx) 6, 2-phenyl-2dihydrooxazine (PhDHOx) 7 and 2-phenyl-2-tetrahydrooxazepine (PhTHOx) 1. All $\mathrm{k}_{\mathrm{p}}$ 's are given in L $\mathrm{mol}^{-1} \mathrm{~s}^{-1}$.

The difference in reactivity between 2-oxazolines and 2-DHOx's is usually explained by the increased steric demand in the $\mathrm{S}_{\mathrm{N}} 2$-type cationic transition state, ${ }^{19}$ related to the ground state 'sofa' or envelope-type conformation of a 2-oxazine ring (Figure 1a). As the additional steric demand of another methylene insertion is expected to be small, the markedly slower polymerization rate of 2-phenyl-2-THOx 1 is surprising. However, an analysis of the ground state conformation of $\mathbf{1}$, as observed in the X-ray structure (see Scheme 3 and Figure 1), revealed a striking stereo-electronic difference of the imino ether moiety in the 2-THOx, which is significantly deplanarized around the oxygen atom attached to the electrophilic carbon atom (torsion angle of $67^{\circ}$ around the $\mathrm{O}$ to $\mathrm{C}=\mathrm{N}$ sigma bond). In both the 2-oxazoline and 2-oxazine-type ground states, the imino ether moiety is much more planar (torsion angles of $0^{\circ}$ and $30^{\circ}$ respectively). Importantly, only a planar conformation readily allows resonance bonding between the oxygen lone pairs and the electron withdrawing imino function, as would be required for facile expulsion of the amide leaving group in its energetically favored planar configuration. In other words, in order to reach the cationic transition state, a planarization around the imino ether is required to efficiently transfer electron density from the oxygen to the iminium cation, which will induce additional ring strain in the reactive conformation of 2-THOx's, thus raising the overall barrier for propagation via a classical stereo-electronic effect. ${ }^{32-34}$ Based on the micro-reversibility principle, the same stereo electronic effect can be used to explain the disfavored sevenmembered ring closure from hydroxy amides in the previous attempts at synthesizing this heterocyclic ring, which were shown in this work to be unfeasible reaction pathways (see Scheme 2). 
In conclusion, we demonstrate the first synthesis of seven-membered 2-THOx leading to the structural reassignment of all previously reported non-fused seven-membered 2-THOx CIEs, which were demonstrated to be the $N$-acylated pyrrolidines instead, misassigned as imino ether isomers. ${ }^{24-29}$ Moreover, we have also investigated the CROP of 2-THOx, and found a significant retardation in the seven-membered CIE polymerization, attributed to a non-coplanar arrangement of the imino ether moiety in this strained unsaturated ring system. Our work opens up the possibility for the exploration of 2-THOx both in asymmetric ligand, drug development and in monomer design.

\section{ASSOCIATED CONTENT}

\section{Supporting Information}

In the supporting information (SI) a comparison of all reassigned putative 2-THOx with the literature data of the related $\mathrm{N}$-acylated pyrrolidones is provided. ${ }^{24-29} \mathrm{In}$ addition copies of ${ }^{1} \mathrm{H}$ and ${ }^{13} \mathrm{C}$ NMR for the phenyl based monomers $(\mathbf{6}, 7$ and $\mathbf{1})$ are provided. The synthesis procedures, polymerization procedures and analysis of all molecules and polymers synthesized in this work are also included in the SI.

\section{AUTHOR INFORMATION}

\section{Corresponding Author}

*johan.winne@ugent.be

*richard.hoogenboom@ugent.be

\section{Author Contributions}

'Both authors contributed equally

\section{Notes}

The authors declare no competing financial interests.

\section{ACKNOWLEDGMENT}

This work was financially supported by the IWT. We acknowledge the help of the NMR expertise center, in particularly Tim Courtin. KVH thanks the Hercules Foundation (project AUGE/11/029 "3D-SPACE: 3D Structural Platform Aiming for Chemical Excellence") and the Special Research Fund (BOF) - UGent (project 01N03217) for funding.

\section{REFERENCES}

(1) Verbraeken, B.; Monnery, B. D.; Lava, K.; Hoogenboom, R. The Chemistry of Poly(2Oxazoline)S. Eur. Polym. J. 2017, 88, 451-469.

(2) Yao, S.; Johannsen, M.; Audrain, H.; Hazell, R. G.; Jørgensen, K. A. Catalytic Asymmetric HeteroDiels-Alder Reactions of Ketones: Chemzymatic Reactions. J. Am. Chem. Soc. 1998, 120 (34), 8599-8605. 
(3) Evans, D. A.; Burgey, C. S.; Kozlowski, M. C.; Tregay, S. W. C2-Symmetric Copper(II) Complexes as Chiral Lewis Acids. Scope and Mechanism of the Catalytic Enantioselective Aldol Additions of Enolsilanes to Pyruvate Esters. J. Am. Chem. Soc. 1999, 121 (4), 686-699.

(4) Evans, D. A.; Willis, M. C.; Johnston, J. N. Catalytic Enantioselective Michael Additions to Unsaturated Ester Derivatives Using Chiral Copper(II) Lewis Acid Complexes. Org. Lett. 1999, 1 (6), 865-868.

(5) Kline, T.; Andersen, N. H.; Harwood, E. A.; Bowman, J.; Malanda, A.; Endsley, S.; Erwin, A. L.; Doyle, M.; Fong, S.; Harris, A. L.; et al. Potent, Novel in Vitro Inhibitors of the Pseudomonas Aeruginosa Deacetylase LpxC. J. Med. Chem. 2002, 45 (14), 3112-3129.

(6) Bode, H. B.; Irschik, H.; Wenzel, S. C.; Reichenbach, H.; Müller, R.; Höfle, G. The Leupyrrins: A Structurally Unique Family of Secondary Metabolites from the Myxobacterium Sorangium Cellulosum\#. J. Nat. Prod. 2003, 66 (9), 1203-1206.

(7) Moraski, G. C.; Chang, M.; Villegas-Estrada, A.; Franzblau, S. G.; Möllmann, U.; Miller, M. J. Structure-activity Relationship of New Anti-Tuberculosis Agents Derived from Oxazoline and Oxazole Benzyl Esters. Eur. J. Med. Chem. 2010, 45 (5), 1703-1716.

(8) Meyers, A. I.; Temple, D. L.; Haidukewych, D.; Mihelich, E. D. Oxazolines. XI. Synthesis of Functionalized Aromatic and Aliphatic Acids. Useful Protecting Group for Carboxylic Acids against Grignard and Hydride Reagents. J. Org. Chem. 1974, 39 (18), 2787-2793.

(9) Guillerm, B.; Monge, S.; Lapinte, V.; Robin, J. J. How to Modulate the Chemical Structure of Polyoxazolines by Appropriate Functionalization. Macromol. Rapid Commun. 2012, 33 (19), $1600-1612$.

(10) Aoi, K.; Okada, M. Polymerization of Oxazolines. Prog. Polym. Sci. 1996, 21 (1), 151-208.

(11) Luxenhofer, R.; Han, Y.; Schulz, A.; Tong, J.; He, Z.; Kabanov, A. V.; Jordan, R. Poly(2Oxazoline)s as Polymer Therapeutics. Macromol. Rapid Commun. 2012, 33 (19), 1613-1631.

(12) Lübtow, M. M.; Hahn, L.; Haider, M. S.; Luxenhofer, R. Drug Specificity, Synergy and Antagonism in Ultrahigh Capacity Poly(2-Oxazoline)/Poly(2-Oxazine) Based Formulations. J. Am. Chem. Soc. 2017, 139 (32), 10980-10983.

(13) Morgese, G.; Cavalli, E.; Rosenboom, J. G.; Zenobi-Wong, M.; Benetti, E. M. Cyclic Polymer Grafts That Lubricate and Protect Damaged Cartilage. Angew. Chemie - Int. Ed. 2018, 57 (6), 16211626.

(14) Barz, M.; Luxenhofer, R.; Zentel, R.; Vicent, M. J. Overcoming the PEG-Addiction: Well-Defined Alternatives to PEG, from Structure-property Relationships to Better Defined Therapeutics. Polym. Chem. 2011, 2 (9), 1900.

(15) Lava, K.; Verbraeken, B.; Hoogenboom, R. Poly(2-Oxazoline)s and Click Chemistry: A Versatile Toolbox toward Multi-Functional Polymers. Eur. Polym. J. 2015, 65, 98-111.

(16) Schmitz, M.; Kuhlmann, M.; Reimann, O.; Hackenberger, C. P. R.; Groll, J. Side-Chain CysteineFunctionalized Poly(2-Oxazoline)s for Multiple Peptide Conjugation by Native Chemical Ligation. 
Biomacromolecules 2015, 16 (4), 1088-1094.

(17) Bloksma, M. M.; Schubert, U. S.; Hoogenboom, R. Poly(Cyclic Imino Ether)s beyond 2Substituted-2-Oxazolines. Macromol. Rapid Commun. 2011, 32 (18), 1419-1441.

(18) Levy, A.; Litt, M. Polymerization of Cyclic Imino Ethers. II. Oxazines. J. Polym. Sci. Part B Polym. Lett. 1967, 5 (9), 881-886.

(19) Saegusa, T.; Kobayashi, S.; Nagura, Y. Isomerization Polymerization of 1,3-Oxazine. II. Kinetic Studies of the Ring-Opening Isomerization Polymerization of Unsubstituted 5,6-Dihydro-4H-1,3Oxazine. Macromolecules 1974, 7 (3), 265-272.

(20) Lübtow, M. M.; Hahn, L.; Haider, M. S.; Luxenhofer, R. Drug Specificity, Synergy and Antagonism in Ultrahigh Capacity Poly(2-Oxazoline)/Poly(2-Oxazine) Based Formulations. J. Am. Chem. Soc. 2017, 139 (32), 10980-10983.

(21) Morgese, G.; Verbraeken, B.; Ramakrishna, S. N.; Gombert, Y.; Cavalli, E.; Rosenboom, J.-G.; Zenobi-Wong, M.; Spencer, N. D.; Hoogenboom, R.; Benetti, E. M. Chemical Design of Non-Ionic Polymer Brushes as Biointerfaces: Poly(2-Oxazine)s Outperform Both Poly(2-Oxazoline)s and PEG. Angew. Chemie Int. Ed. 2018, 57 (36), 11667-11672.

(22) Lorson, T.; Jaksch, S.; Lübtow, M. M.; Jüngst, T.; Groll, J.; Lühmann, T.; Luxenhofer, R. A Thermogelling Supramolecular Hydrogel with Sponge-Like Morphology as a Cytocompatible Bioink. Biomacromolecules 2017, 18 (7), 2161-2171.

(23) Miyamoto, M.; Aoi, K.; Saegusa, T. Ring-Opening Polymerization of Six- and Seven-Membered Cyclic Pseudoureas. J. Polym. Sci. Part A Polym. Chem. 1996, 35 (5), 933-945.

(24) Petersson, M. J.; Jenkins, I. D.; Loughlin, W. A. The Use of Phosphonium Anhydrides for the Synthesis of 2-Oxazolines $\{$,$\} 2-Thiazolines and 2-Dihydrooxazine under Mild Conditions. Org.$ Biomol. Chem. 2009, 7 (4), 739-746.

(25) Pouliot, M.-F.; Angers, L.; Hamel, J.-D.; Paquin, J.-F. Synthesis of 2-Oxazolines and Related NContaining Heterocycles Using [Et2NSF2]BF4 as a Cyclodehydration Agent. Tetrahedron Lett. 2012, 53 (32), 4121-4123.

(26) Reddy, D. N.; Thirupathi, R.; Tumminakatti, S.; Prabhakaran, E. N. A Method for Stabilizing the Cis Prolyl Peptide Bond: Influence of an Unusual $\mathrm{N} \rightarrow \pi *$ Interaction in 1,3-Oxazine and 1,3Thiazine Containing Peptidomimetics. Tetrahedron Lett. 2012, 53 (33), 4413-4417.

(27) Schweiker, S. S.; Loughlin, W. A.; Lohning, A. S.; Petersson, M. J.; Jenkins, I. D. Synthesis, Screening and Docking of Small Heterocycles as Glycogen Phosphorylase Inhibitors. Eur. J. Med. Chem. 2014, 84, 584-594.

(28) Alhalib, A.; Kamouka, S.; Moran, W. J. Iodoarene-Catalyzed Cyclizations of Unsaturated Amides. Org. Lett. 2015, 17 (6), 1453-1456.

(29) Mollo, M. C.; Orelli, L. R. Microwave-Assisted Synthesis of 2-Aryl-2-Oxazolines, 5,6-Dihydro4H-1,3-Oxazines, and 4,5,6,7-Tetrahydro-1,3-Oxazepines. Org. Lett. 2016, 18 (23), 6116-6119.

(30) Hall, H. K.; Schneider, A. K. Polymerization of Cyclic Esters, Urethans, Ureas and Imides. J. Am. 
Chem. Soc. 1958, 80 (23), 6409-6412.

(31) Kušan, J.; Keul, H.; Höcker, H. Cationic Ring-Opening Polymerization of Tetramethylene Urethane. Macromolecules 2001, 34 (3), 389-395.

(32) Deslongchamps, P.; Lebreux, C.; Taillefer, R. The Importance of Conformation of the Tetrahedral Intermediate in the Hydrolysis of Amides. Selective Cleavage of the Tetrahedral Intermediate Controlled by Orbital Orientation. Can. J. Chem. 1973, 51 (10), 1665-1669.

(33) Deslongchamps, P.; Dubé, S.; Lebreux, C.; Patterson, D. R.; Taillefer, R. J. The Hydrolysis of Imidate Salts. Stereoelectronic Control in the Cleavage of the Hemiorthoamide Tetrahedral Intermediate. Can. J. Chem. 1975, 53 (18), 2791-2807.

(34) Deslongchamps, P.; Cheriyan, U. O.; Taillefer, R. J. Hydrolysis of Cyclic Unsymmetrical Anti Imidate Salts. New Evidence for Stereoelectronic Control. Can. J. Chem. 1979, 57 (24), 32623271.

Insert Table of Contents artwork here

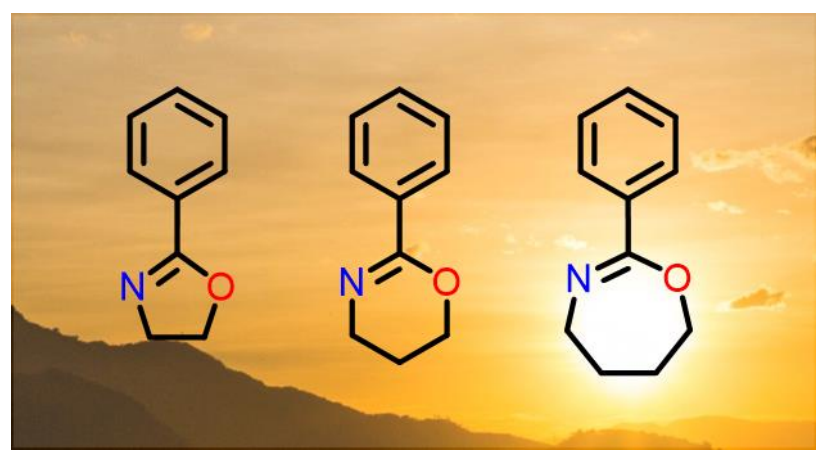

